\title{
Ehrenfest time and the coherent backscattering off ballistic cavities
}

\author{
Saar Rahav and Piet W. Brouwer \\ Laboratory of Atomic and Solid State Physics, Cornell University, Ithaca 14853, USA.
}

(Dated: August 26, 2018)

\begin{abstract}
If the Ehrenfest time $\tau_{\mathrm{E}}$ of a ballistic cavity is not negligible in comparison to its dwell time $\tau_{\mathrm{D}}$, the weak localization correction to the cavity's transmission is suppressed proportional to $\exp \left(-\tau_{\mathrm{E}} / \tau_{\mathrm{D}}\right)$. At the same time, quantum interference enhances the probability of reflection into the mode of incidence by a factor two. This 'enhanced backscattering' does not depend on the Ehrenfest time. We show that, in addition to the diagonal enhanced backscattering, there are off-diagonal contributions to coherent backscattering that become relevant if $\tau_{\mathrm{E}} \gtrsim \tau_{\mathrm{D}}$.
\end{abstract}

PACS numbers: 73.23.-b,05.45.Mt,05.45.Pq,73.20.Fz

Weak localization and enhanced backscattering are two signatures of quantum interference in disordered or chaotic conductors [1, 2]. Both refer to transmission and reflection averaged over an ensemble, e.g., obtained by slight variations of the sample shape. Weak localization is a small negative interference correction to the transmission; Enhanced backscattering is the phenomenon that the probability of reflection into the incident scattering mode is twice the probability of reflection into a different mode. Both weak localization and enhanced backscattering require the presence of time-reversal symmetry. In this letter we consider these phenomena for a twodimensional cavity or 'quantum dot' with ballistic and chaotic classical dynamics. For ballistic cavities the underlying classical dynamics is known to play an important role determining the quantum interference effects [3].

Generally, weak localization and enhanced backscattering are very closely related phenomena. There is a particularly simple argument making this point for the case of a chaotic cavity considered here [4]. The chaotic dynamics is taken to imply that averages of the squares of elements of the cavity's transmission and reflection matrices $t$ and $r$ are equal, except for the squares of the diagonal elements of $r$, which are a factor two larger because of enhanced backscattering [5, 6, 7],

$$
\left\langle\left|r_{n n}\right|^{2}\right\rangle=2\left\langle\left|r_{m n}\right|^{2}\right\rangle=2\left\langle\left|t_{k n}\right|^{2}\right\rangle, \quad \text { if } m \neq n .
$$

Here $n$ and $m$, which label modes in the left contact, can take the values $n, m=1,2, \ldots, N_{1}$, where $N_{1}$ is the number of propagating modes in that contact, and $k$ takes the values $k=1, \ldots, N_{2}$, where $N_{2}$ is the number of modes in the right contact. Since unitarity gives

$$
\sum_{m=1}^{N_{1}}\left|r_{m n}\right|^{2}+\sum_{k=1}^{N_{2}}\left|t_{k n}\right|^{2}=1,
$$

one finds that the average of the square of a transmission matrix element is

$$
\left\langle\left|t_{k n}\right|^{2}\right\rangle=\frac{1}{N+1}, \quad N=N_{1}+N_{2}
$$

The amount by which $\left\langle\left|t_{k n}\right|^{2}\right\rangle$ is smaller than $1 / N$ is the weak localization correction.
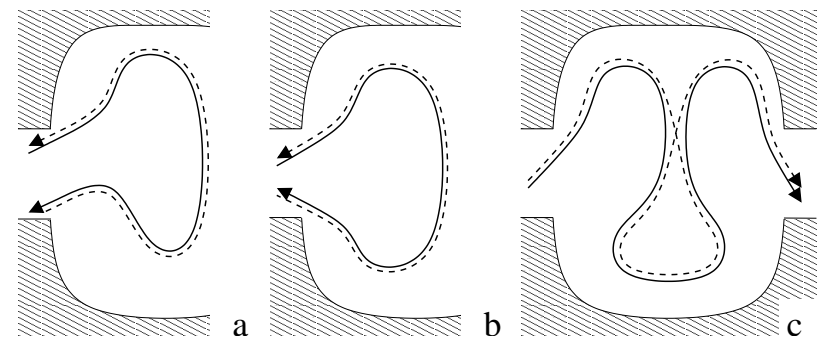

FIG. 1: Schematic drawing of pairs of classical trajectories contribution to coherent backscattering ( $\mathrm{a}$ and $\mathrm{b}$ ) and weak localization (c).

In order to understand the semiclassical origin of enhanced backscattering, mere inspection of the semiclassical formula for the square of a reflection matrix element $\left|r_{m n}\right|^{2}$ is sufficient. Indeed, one has [8]

$$
\left|r_{m n}\right|^{2}=\frac{1}{2 N_{1}} \sum_{\alpha, \beta} \frac{1}{M_{\alpha} M_{\beta}^{*}} e^{i\left(\mathcal{S}_{\alpha}-\mathcal{S}_{\beta}\right) / \hbar},
$$

where $\alpha$ and $\beta$ denote classical trajectories that connect the left contact to itself, start with initial transverse momentum compatible with mode $n$, and end with transverse momentum compatible with mode $m, \mathcal{S}$ is the classical action (to be specified later), and $M_{\alpha}$ and $M_{\beta}$ are stability amplitudes. The modes in each contact have quantized transverse momentum

$$
p_{\perp}(m)= \pm \pi \hbar m / W_{j}, \quad m=1, \ldots, N_{j},
$$

where $W_{j}$ is the width of the contact and the subscript $j=1,2$ refers to the left and right contacts, respectively. Enhanced backscattering then follows from the simple observation that whereas generically only contributions $\alpha=\beta$ contribute to $\left\langle\left|r_{n m}\right|^{2}\right\rangle$ if $n \neq m,\left\langle\left|r_{n n}\right|^{2}\right\rangle$ also has contributions from $\alpha$ and $\beta$ being time-reversed trajectories [5, 6, 7]. Examples of pairs of time-reversed trajectories are shown in Fig. 目 and b. Since actions and stability amplitudes are invariant under time reversal, one immediately arrives at the first equality in Eq. (11).

The semiclassical theory of weak localization is more complicated than this. As was first shown by Aleiner and 
Larkin [9], weak localization in ballistic cavities is rooted in destructive interference of two trajectories that differ only near a small-angle self-encounter, where one trajectory intersects itself and the other trajectory does not, see Fig. 11. Since the two trajectories are close everywhere in phase space (up to time reversal), their action difference is small. Aleiner and Larkin's original theory was formulated in a language borrowed from the theory of disordered conductors; Richter and Sieber showed that the same structure translates to the trajectory-based picture of Eq. (4) 10] (see also Ref. 11).

Aleiner and Larkin's theory of weak localization in ballistic cavities not only solved a problem, it also pointed to a new one: Since weak localization in a ballistic cavity involves the exponential divergence and convergence of trajectories, the complete theory of weak localization requires knowledge of the Lyapunov exponent $\lambda$ of the classical dynamics in the cavity [9]. The Lyapunov exponent enters the weak localization correction through the Ehrenfest time $\tau_{\mathrm{E}}$, which is the time it takes for two classical trajectories initially separated by a phase space distance $\sim \hbar$ to diverge and be separated by a classical phase space distance [12],

$$
\tau_{\mathrm{E}}=\frac{1}{\lambda} \ln N+\text { const, }
$$

where the added term does not scale with Planck's constant. (The channel number $N$ is proportional to $\hbar^{-1}$.) Since a small-angle encounter contributing to weak localization has a duration $\sim \tau_{\mathrm{E}}$, the weak localization correction is proportional to $\exp \left(-\tau_{\mathrm{E}} / \tau_{\mathrm{D}}\right)$, where $\tau_{\mathrm{D}}$ is the cavity's mean dwell time [9, 13, 14]. Enhanced backscattering, on the other hand, involves no small-angle intersections, hence it has no dependence on $\tau_{\mathrm{E}}$.

Both results, exponential suppression of weak localization with increasing Ehrenfest time and the Ehrenfesttime independence of the factor two enhancement of $\left\langle\left|r_{n n}\right|^{2}\right\rangle$ over generic off-diagonal reflection coefficients $\left\langle\left|r_{n m}\right|^{2}\right\rangle$, have a solid foundation, in semiclassical theory [5, 6, 9, 13, 14] as well as numerical simulations 14, 15]. How can they be reconciled?

The answer to the puzzle, which will be derived below, is that coherent backscattering involves not only the factor-two enhancement of averaged diagonal reflection coefficients, but also a slight reduction of off-diagonal elements of the reflection matrix if $\tau_{\mathrm{E}} \gtrsim \tau_{\mathrm{D}}$. For the off-diagonal elements we find, to leading order in $1 / N$,

$$
\left\langle\left|r_{m n}\right|^{2}\right\rangle=\frac{1}{N}\left(1-\frac{1}{2 \lambda \tau_{\mathrm{D}}|m-n|^{1+1 / \lambda \tau_{\mathrm{D}}}}\right) .
$$

The reduction of near-diagonal elements in Eq. (77) explains why the simple connection between weak localization and enhanced backscattering used to derive Eq. (3) above fails if $\tau_{\mathrm{E}}$ is not negligible. One verifies that summation over all off-diagonal reflection matrix elements

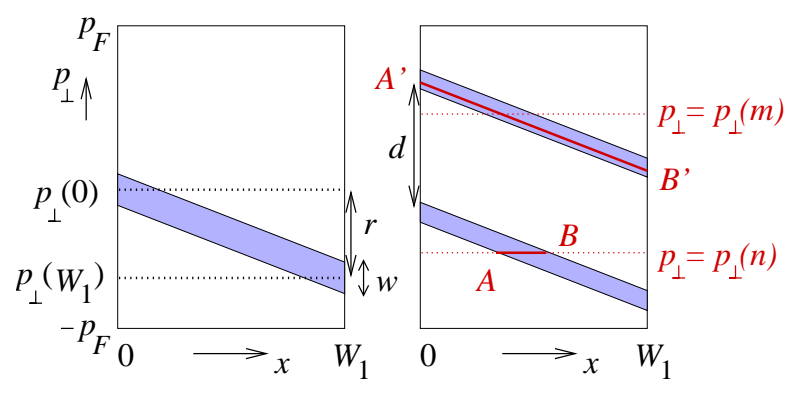

FIG. 2: Left: the surface of section of a band, together with the definitions of the band's center momentum, momentum range $r$, and momentum width $w$. The maximal transverse momentum is the Fermi momentum $p_{F}$. Right: a band and its image. The image band is the surface of section of the trajectories in the band upon exit, with the transverse momentum $p_{\perp}$ reversed.

gives a contribution $\left[-1+\exp \left(-\tau_{\mathrm{E}} / \tau_{\mathrm{D}}\right)\right] / N$ to the total reflection probability, which precisely replaces the $\tau_{\mathrm{E}^{-}}$ independent interference correction from $\left\langle\left|r_{n n}\right|^{2}\right\rangle$ with the same $\tau_{\mathrm{E}}$-dependence as the weak localization correction [14, 16]. In Eq. (7) we omitted terms that decay proportional to $|m-n|^{-2}$ or faster and have no net contribution to the total reflection. The full expression for $\left\langle\left|r_{n m}\right|^{2}\right\rangle$ is obtained by adding Eqs. (12), (14), and (16) below.

We now describe the details of our calculation. In order to obtain simple, non system-specific results, we examine the chaotic cavity in the limit $N \rightarrow \infty$ (i.e., $\hbar \rightarrow 0$ ), while keeping $\tau_{\mathrm{E}} / \tau_{\mathrm{D}}$ fixed. In this limit, the widths of the contacts scale to zero, $W_{j} \propto 1 / \ln N, j=1,2$. The classical trajectories entering the cavity through the left contact are indexed with the help of their transverse momentum $p_{\perp}$ and the coordinate $x$ along the contact's cross section. Alternatively, trajectories exiting the cavity are indexed with $x$ and minus their transverse momentum. On this 'Poincaré surface of section', trajectories appear in 'bands' that remain close in phase space throughout the passage through the cavity [17, 18]. The phase space points that belong to a band are concentrated along stable manifolds at entry and unstable manifolds at exit. The band edges are determined by the contact edges. Since the widths $W_{1}$ and $W_{2}$ scale to zero in the classical limit taken here, we can linearize the dynamics within each band. While trajectories inside a band have correlated actions, we assume that actions of trajectories in different bands are uncorrelated. (This precludes a theory of weak localization.) Linearization of the classical dynamics inside a band also means that the edges of nearby bands are parallel lines in the Poincaré surface of section. For simplicity, we assume that all relevant bands are parallel; we neglect 'folds', bands with a strong curvature that do not span the entire range $0<x<W_{1}$.

For each band in the Poincaré surface of section referencing the trajectories at their entrance into the cavity, there exists an 'image band' in the Poincaré surface of 
section referencing the trajectories at their exit. This 'entrance-exit' relation maps the Poincaré surface of section onto itself, so that one obtains a connection between pairs of time-reversed bands. (Although bands may be mapped onto themselves, only pairs of different bands can give rise to interference.)

Each band is characterized by its center momentum $p(0)$ at $x=0$, its center momentum $p\left(W_{1}\right)$ at $x=W_{1}$ and its momentum width $w$, see Fig. 2] (Note that it is implicitly assumed that the cross section is at the lead opening.) For a band, we define its momentum range as $r=\left|p(0)-p\left(W_{1}\right)\right|$. For a pair of bands, we define their distance $d$ as the difference of the center momenta. Both bands in a pair have the same width [18]. Since trajectories originating from bands a distance $d$ apart are correlated for a time $\lambda^{-1} \ln p_{F} / d$ 9, 12], the dwell time $t$ for trajectories in a band a distance $d$ from its image is at least $t_{\mathrm{min}}$, with

$$
t_{\min }=(2 / \lambda) \ln \left(p_{F} / d\right),
$$

where $p_{F}$ is the Fermi momentum. As the momentum width $w$ of a band scales $\propto \exp (-\lambda t)$ [17], we have $w \ll d, r$ for a typical pair of bands and for dwell times of order $\tau_{\mathrm{D}}$ and larger. This allows us to neglect the momentum width of bands $w$ with respect their distance $d$ or momentum range $r$ in the following considerations.

It is important to note that in each band there is at most one trajectory with given transverse momenta for entrance and exit. This is illustrated in the right panel of Fig. 2 A trajectory which starts with transverse momentum $p_{\perp}=p_{\perp}(n)$ starts somewhere on the linear segment $\mathrm{AB}$ in the figure, which is the intersection of the band and the line $p_{\perp}=p_{\perp}(n)$. Since the phase space points $\mathrm{A}$ and $\mathrm{B}$ correspond to the band's edge, they are mapped to the edges $x=0$ and $x=W_{1}$ of the left contact upon exit: The image of the segment $A B$ is the line $\mathrm{A}^{\prime} \mathrm{B}$ ' in the right panel of Fig. 2] For an (inverted) outgoing transverse momentum $p_{\perp}(m)$ there is at most one intersection with A'B'. Note that there is precisely one solution if $p_{\perp}(m)$ is in the momentum range of the image band and if we may ignore the possibility that the lines $p_{\perp}=p_{\perp}(n)$ or $p_{\perp}=p_{\perp}(m)$ cut the bands close to $x=0$ or $x=W_{1}$. This simplification is allowed since in the limit considered here all bands are narrow, see the discussion following Eq. (8).

Having found a way to index families of trajectories appearing in the semiclassical formula (4), we now describe the calculation of the contribution of such trajectories to $\left\langle\left|r_{m n}\right|^{2}\right\rangle$. Hereto, we need to specify the action $\mathcal{S}$ entering into Eq. (44) [8],

$$
\mathcal{S}=\tilde{\mathcal{S}}+p_{\perp} x-p_{\perp}^{\prime} x^{\prime}
$$

where $\tilde{\mathcal{S}}$ is the standard coordinate-dependent classical action [8] and unprimed and primed variables refer to entrance and exit, respectively. The last two terms in
Eq. (9) are obtained from the lead wave functions and cause $\mathcal{S}$ to depend explicitly on the precise location of the contacts. In the linearized formulation used here, the stability amplitude $M$ is the same for all trajectories in a band. Neglecting the band width $w$ with respect to its momentum range $r,|M|^{2}$ can be estimated as

$$
|M|^{2}=r^{2} / p_{F} w .
$$

Before calculating the quantum-interference corrections from pairs of time-reversed bands, we calculate the leading classical contribution to $\left\langle\left|r_{m n}\right|^{2}\right\rangle$ using the band picture. The classical contribution to $\left\langle\left|r_{m n}\right|^{2}\right\rangle$ follows from taking $\alpha=\beta$ in Eq. (44). In order to find $\left\langle\left|r_{m n}\right|^{2}\right\rangle$ we need to sum over over all reflection bands for which the initial momentum range contains $p_{\perp}=\pi \hbar n / W_{1}$ or $p_{\perp}=-\pi \hbar n / W_{1}$ and for which the image band contains $p_{\perp}=\pi \hbar m / W_{1}$ or $-\pi \hbar m / W_{1}$. The number density $n(t)$ of bands with this property and with dwell time $t$ is obtained by multiplying the probability density $\left(N_{1} / N \tau_{\mathrm{D}}\right) \exp \left(-t / \tau_{\mathrm{D}}\right)$ for escape at time $t$ through contact 1 by the probability $\left(r / p_{F}\right)^{2}$ that the band and the image band intersect one of the lines $p_{\perp}= \pm \pi \hbar n / W_{1}$ and $p_{\perp}= \pm \pi \hbar m / W_{1}$, respectively, and dividing by the area fraction of one band, $w / 2 p_{F}$,

$$
n(t)=\frac{2 N_{1} r^{2}}{N w p_{F} \tau_{\mathrm{D}}} e^{-t / \tau_{\mathrm{D}}} .
$$

Dividing $n(t)$ by $2 N_{1}|M|^{2}$ and integrating over time, we find the well-known leading-order result

$$
\left\langle\left|r_{m n}\right|^{2}\right\rangle=1 / N
$$

There are two distinct types of coherent backscattering corrections to Eq. (12): From trajectories which start and end with approximately opposite transverse momenta (as in Fig. (1) and from trajectories for which incoming and outgoing transverse momenta are approximately equal (as in Fig. 1b). We address the former contribution first.

In order to have an interference contribution from trajectories with approximately opposite incoming and outgoing momenta, the momentum distance $d$ of their band and image band must be less than the range $r$ of each band: only then there exist values of the incoming and outgoing momenta that intersect both bands. (Since we take bands to be parallel, both have the same momentum range $r$.) For each pair of bands, there are two trajectories with incoming transverse momentum $\pm \pi n \hbar / W_{1}$ and outgoing transverse momentum $\mp \pi m \hbar / W_{1}$. If $n=m$ these two trajectories are time reversed and have the same action $\mathcal{S}$. Their interference gives half the standard enhancement of $\left\langle\left|r_{n n}\right|^{2}\right\rangle$. (The other half comes from the class of trajectories considered next.) If $n \neq m$ the action difference is calculated to be

$$
\Delta \mathcal{S}=\pi \hbar(m-n) r^{-1} d .
$$


In calculating Eq. (13) we used the linearity of the band and neglected the width of a band, $w$, in comparison to $d$ and $r$. The sign of the action difference is irrelevant, since the sum over trajectories (4) contains both $\Delta \mathcal{S}$ and $-\Delta \mathcal{S}$. In order to find the corresponding interference contribution $\delta_{|m-n|}$ to $\left\langle\left|r_{n m}\right|^{2}\right\rangle$, we need the number density $n(t, d)$ of reflection bands that have distance $d$ and intersect both $p_{\perp}= \pm n \pi \hbar / W_{1}$ and $p_{\perp}= \pm m \pi \hbar / W_{1}$,

$$
n(t, d)=\frac{N_{1}\left(r-|m-n| \hbar \pi / W_{1}-d\right)}{N w p_{F} \tau_{\mathrm{D}}} e^{-\left(t-t_{\min } / 2\right) / \tau_{\mathrm{D}}},
$$

if $0<d<r-|m-n| \hbar \pi / W_{1}$ and $t>t_{\min }(d) ; n(t, d)=0$ otherwise. Both the minimum dwell time $t_{\text {min }}$ and the exponential enhancement factor $\exp \left(t_{\min } / 2\right)$ follow from the correlations of trajectories in nearby bands, see Eq. (8) and Refs. 11 and 14. Integrating over the dwell time $t$ and the band distance $d$, we then find

$$
\delta_{l}=\int_{0}^{l-y(l)} d z \frac{l-y(l)-z}{l^{2} N}(z / l)^{1 / \lambda \tau_{\mathrm{D}}} \cos (\pi z),
$$

where $y(l)=l^{2} \pi \hbar / W_{1} r$.

Contributions of trajectories for which the incoming and outgoing transverse momenta are approximately equal are treated similarly. The main difference with the preceding contribution is that, in this case, small action differences are found for pairs of bands that are typically far from each other. The action difference can be estimated in exactly the same way as it was done previously,

$$
\Delta \mathcal{S}=\pi \hbar(m-n)\left(p+p^{\prime}\right) r^{-1},
$$

where $p$ and $p^{\prime}$ are the center momenta of the two bands in the pair taken at $x=0$ or $x=W$. We then find that the interference correction to $\left\langle\left|r_{n m}\right|^{2}\right\rangle$ from this class of trajectories is $\delta_{|m-n|}^{\prime}$, with [19]

$$
\delta_{l}^{\prime}=\int_{y(l)}^{l} d z \frac{z-y(l)}{l^{2} N} \cos (\pi z) .
$$

Equations (14) and (16) contain the main quantitative result of this letter. Summation over $l$ gives the coherent backscattering correction to the total reflection [14], which now depends on the Ehrenfest time in the same way as the weak localization correction and, together with weak localization, preserves the unitarity relation (2). Combining both interference contributions and evaluating the integrals in the limit $\hbar \rightarrow 0$ and for large $\lambda \tau_{\mathrm{D}}$ one finds Eq. (77) above with the extra condition that $|m-n|$ be small in comparison to $r W_{1} / \hbar$, plus a contribution that decays faster with $l$ than Eq. (7) and sums to zero if summed over $l$ in the limit $\hbar \rightarrow 0$ at fixed $\tau_{\mathrm{E}} / \tau_{\mathrm{D}}$. While Eqs. (14) and (16) were derived under the assumption that all reflection bands are parallel in the Poincaré surface of section, we verified that relaxing this assumption for Eq. (16), where it is most questionable, only gives corrections to $\delta_{l}^{\prime}$ that are of order $1 / N^{2}$ and sum to zero in the limit $\hbar \rightarrow 0$ at fixed $\tau_{\mathrm{E}} / \tau_{\mathrm{D}}$.

In conclusion, we have shown that there are offdiagonal contributions to coherent backscattering off a ballistic chaotic cavity which are essential if the Ehrenfest time $\tau_{\mathrm{E}}$ is not small in comparison to the dwell time $\tau_{\mathrm{D}}$. To the best of our knowledge, the Ehrenfest-time dependence of the average reflection coefficients $\left\langle\left|r_{n m}\right|^{2}\right\rangle$ is the first manifestation of the Ehrenfest-time in a quantity that does not involve a summation over lead modes. As such, coherent backscattering not only probes the Ehrenfest regime for ballistic electronic transport, it also provides a method to access the Ehrenfest regime for experiments with classical waves, such as microwave billiards.

We thank Carlo Beenakker, Douglas Stone, and Denis Ullmo for stimulating discussions. The unpublished Ref. 15 motivated us to start this research. Upon completion of this work Refs. 16 and 20 appeared on the cond-mat archive, in which the off-diagonal nature of coherent backscattering was also pointed out. We thank Philippe Jacquod for sending us a preprint of Ref. 16. This work was supported by the NSF under grant no. DMR 0334499 and by the Packard Foundation.

[1] Mesoscopic Quantum Physics, edited by E. Akkermans, G. Montambaux, J.-L. Pichard, and J. Zinn-Justin (North-Holland, 1995).

[2] C. W. J. Beenakker, Rev. Mod. Phys. 69, 731 (1997).

[3] See, e.g., A. D. Stone in Ref. 1

[4] N. Argaman, Phys. Rev. Lett. 75, 2750 (1995).

[5] E. Doron, U. Smilansky, and A. Frenkel, Physica D 50, 367 (1991).

[6] C. H. Lewenkopf and H. A. Weidenmüller, Ann. Phys. 212, 53 (1991).

[7] H. U. Baranger, R. A. Jalabert, and A. D. Stone, Phys. Rev. Lett. 70, 3876 (1993).

[8] R. A. Jalabert, H. U. Baranger, and A. D. Stone, Phys. Rev. Lett. 65, 2442 (1990).

[9] I. L. Aleiner and A. I. Larkin, Phys. Rev. B 54, 14423 (1996).

[10] K. Richter and M. Sieber, Phys. Rev. Lett. 89, 206801 (2002).

[11] S. Heusler, S. Müller, P. Braun, and F. Haake, Phys. Rev. Lett. 96, 066804 (2006).

[12] G. M. Zaslavsky, Phys. Rep. 80, 157 (1981).

[13] I. Adagideli, Phys. Rev. B 68, 233308 (2003).

[14] S. Rahav and P. W. Brouwer, Phys. Rev. Lett. 95, 056806 (2005); Phys. Rev. B 73, 035324 (2006).

[15] A. Tajic, J. Tworzydlo, and C. W. J. Beenakker, condmat/0406271, version 1 .

[16] Ph. Jacquod and R. S. Whitney, Phys. Rev. B, 73195115 (2006).

[17] L. Wirtz, J.-Z. Tang, and J. Burgdörfer, Phys. Rev. B 59, 2956 (1999).

[18] J. Tworzydlo, A. Tajic, H. Schomerus, and C. W. J. Beenakker, Phys. Rev. B 68, 115313 (2003).

[19] The main contribution of Eq. (16) to the total reflection, though negligible in the classical limit taken here, is from pairs of bands with $p+p^{\prime} \approx 0$ or $p+p^{\prime} \approx \pm 2 r$. This describes the effect of diffraction at the contact edges. 
[20] R. S. Whitney and Ph. Jacquod, Phys. Rev. Lett., 96, 206804 (2006). 